\title{
Properties of gelatins from skins of fish-black tilapia (Oreochromis mossambicus) and red tilapia (Oreochromis nilotica)
}

\begin{abstract}
Fish skins are potential sources of gelatin. Therefore a study on the extraction and determination of the physicochemical characteristics of gelatin obtained from the skins of black (Oreochromis mossambicus) and red (Oreochromis nilotica) tilapia was undertaken. The extraction was carried out by a series of steps involving washings with $0.2 \%(\mathrm{w} / \mathrm{v})$ sodium hydroxide and sulfuric acid, and $1.0 \%(\mathrm{w} / \mathrm{v})$ of citric acid. This was followed by a final extraction with water at $45{ }^{\circ} \mathrm{C}$ for $12 \mathrm{~h}$ and the colloidal suspension was freeze-dried. Visual appearance, odour, $\mathrm{pH}$, bloom strength, viscosity, melting point and amino acid profile of the gelatins were evaluated. The gelatins from both the black and the red tilapias were snowy white, shiny and light-textured in appearance. The gelatin of black tilapia skin had a strong fishy odour while that of the red tilapia skin had a barely detectable odour. Their $\mathrm{pH}$ values were in the vicinity of 3 . The bloom strength of gelatin from black tilapia skin was higher $(180.8 \mathrm{~g})$ than that from red tilapia skin (128.1 g). The black tilapia skin gelatin was also significantly more viscous, had a higher melting point, and had a higher total amino acid content.
\end{abstract}

Keyword: Fish gelatin; Tilapia; Physicochemical characteristics 